\title{
Cadence tracking and disturbance rejection in FES cycling for paraplegic subjects: a case study
}

\author{
Lucas O. da Fonseca, Antônio P. L. Bó, Juliana A. Guimarães, Miguel E. G. Paredes, \\ Ulisses de Araújo, Christine Azevedo-Coste and Emerson Fachin-Martins
}

\begin{abstract}
Functional Electrical Stimulation cycling has numerous benefits for subjects with Spinal Cord Injury. It can improve cardiovascular function, increase muscular mass, and reduce bone mass loss. However, some limitations, e.g. lack of optimal control strategies that would delay fatigue, may still prevent this technology from achieving its full potential. This work tests a control strategy on a complete spinal cord injury subject with a stationary tadpole trike. Two experiments were performed: reference tracking and disturbance rejection. The results show that reference tracking is possible above the cadence of $25 \mathrm{rpm}$ with a mean absolute error lower than 2.5 rpm. The disturbance test showed that it may cause the cadence to drop but still maintain movement if it doesn't get below 25 $\mathrm{rpm}$. When the disturbance is removed, the system is capable of returning the cadence to the initial value.
\end{abstract}

\section{INTRODUCTION}

Several studies have presented evidence of benefits that cycling assisted by Functional Electrical Stimulation (FES) may provide to subjects with Spinal Cord Injury (SCI) $[1,2$, 3]. Among the observed improvements, of particular relevance are increased muscular mass, enhanced cardiovascular function, and reduced bone mass loss.

Nevertheless, some limitations within the current technology may still prevent further benefits to the user. For instance, studies have shown that power output obtained using electrical stimulation is reduced when compared to the levels reached using volitional recruitment [4]. Also, the accelerated fatigue induced by FES may prevent achieving higher cardiovascular function improvement.

Those limitations are often associated to features related to motor unit recruitment using surface electrical stimulation. In this scenario, we believe improvements in overall performance may be achieved by applying innovative techniques, such as electrode arrays and asynchronous stimulation [5], and complementary control strategies that will enable generation of smooth cycling power patterns.

In our work, the general goal is to investigate new stimulation and control strategies that may improve the overall performance, both in terms of delivered power and fatigue reduction. In a previous work [6], we have presented results on cycling cadence regulation on healthy subjects. Here, based on similar setup and controller, we present a case study involving one paraplegic subject, as well as results for trajectory tracking and disturbance rejection.

The paper is organized as follows. The next section describes the method, including both hardware and software development, as well as the participating subject and protocol.

* Research partially supported by CAPES, CNPq, INRIA and FAPDF.

L. O. da Fonseca, Antônio P. L. Bó, J. A. Guimarães, Miguel E. G. Paredes, and Emerson Fachin-Martins are with the University of Brasília, Brasília, Brazil (e-mail: lucasfonseca@lara.unb.br). Ulisses de Araújo is with CETEFE, Brasília, Brazil. C. Azevedo-Coste is with CAMIN, LIRMM/INRIA, Montpellier, France.
On the following section the results are presented, as well as the corresponding discussion. Finally, the last section presents the concluding remarks and future works.

\section{METHOD}

This experimental case study is based on a recumbent tadpole trike adapted for use by paraplegic subjects. The experimental setup, control algorithm, participating subject, and protocol are described below.

\section{A. Experimental setup}

The setup was based on an adapted tadpole trike (HP3, Brazil), depicted in Figure 1. The tadpole trike, which features two wheels at the front and one at the back, was chosen for its improved stability when compared to delta tricycles. Among the modifications required for use by paraplegic subjects, supporting surfaces for the whole foot have been attached to each pedal. Also, a vertical rod was rigidly fixed to this structure and the leg in order to secure the limb and prevent residual movements outside the sagittal plane. This structure also kept the ankle joint at $90^{\circ}$ at all times.

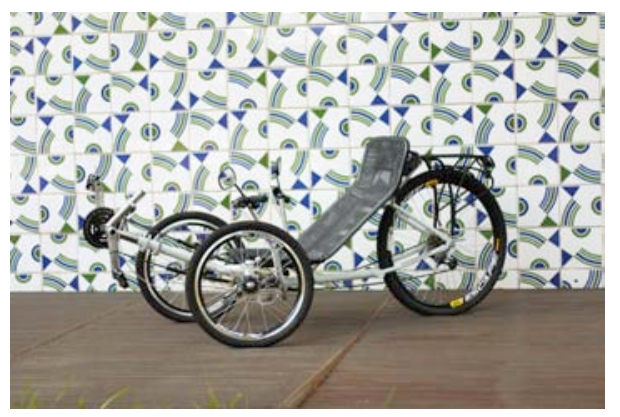

Figure 1. The tadpole Trike used in this work.

In addition to the trike itself, a passive cycling trainer was employed to enable indoor and stationary trials. A mechanical assembly was attached to enable instantaneous application of load disturbances to the trainer. As illustrated in Figure 2, increasingly strong disturbances may be applied by using heavier loads.

The electrical stimulation was applied to the quadriceps muscle groups in order to achieve lower limb extension. There was no way to actively induce flexion. However, due to

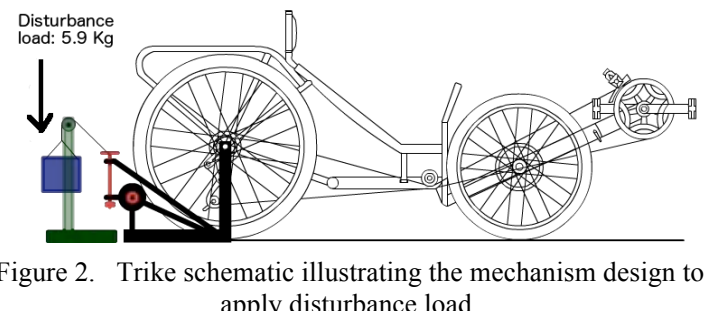


the fixed circular shape of the pedals' trajectory, the extension of one leg led to the flexion of the order, thanks to the bilateral stimulation synchronous timing that was applied.

As for the stimulation unit, a Rehastim (Hasomed, Germany) stimulator was employed. It's an 8-channel current-controlled stimulator that outputs biphasic square pulses. The stimulation parameters (frequency, amplitude, and pulse width) can be updated online through a USB connection. Control algorithms and additional processing are implemented in Python and executed in a personal computer at $25 \mathrm{~Hz}$. The controller receives crank position and angular speed readings from wireless inertial sensors (3space, Yei Technology, United States). The sensing unit is composed of 3 -axis accelerometers, gyrometers and magnetometers, and provides measurements of crank position and angular speed based on fusion of data acquired from those individual sensors.

\section{B. Control strategy}

Closed-loop control of stimulation pulses is performed using two control loops. As summarized by the diagram depicted in Figure 3, stimulation phases are computed based on crank position and cadence.

Stimulation profile for each pedaling revolution is determined within the inner loop. Computation is defined based on static biomechanical properties of recumbent cycling position with respect to the crank set. Furthermore, stimulation patterns are then adjusted based on cycling cadence, similar to what was proposed by [7], to take into account both the artificial and natural delays involved. In order to accomplish that, the system shifts the angular positions in which the stimuli are applied. The faster the cadence, the earlier the stimuli must take place and stop.

Furthermore, stimulation intensity (modulated using pulse width in this work) is controlled in order to provide automatic capability to adjust FES level for the required cycling cadence. The goal while providing this functionality is to improve system robustness with respect to different nominal stimulation amplitudes selected within the initialization procedure, which remain constant for the entire duration of the experiment. Also, such control action will react for changes in muscle response, e.g. FES induced fatigue. On this case study, a PI controller is used based on the following parameters: $K_{P}=10^{-2}$, and $K_{I}=10^{-5}$. Further details on the overall controller are provided in [6].

\section{Subjects and protocol}

After clarification and signing the ethics committee informed consent (CAAE 50337215.1.0000.0030, approval number 1.413 .934 , February, $18^{\text {th }} 2016$ ), one participant took part in this case study. The volunteer (age: 37 years; height: $1.70 \mathrm{~m}$; body mass: $67.40 \mathrm{Kg}$ ) has a complete sensory-motor thoracic traumatic spinal cord injury (T9; AIS A - American Spinal Injury Association Impairment Scale) for 3 years by the time of this work. $\mathrm{He}$ is an active athlete, competing on sailing and rowing. Before the tests described here, the subject underwent five months of "pre-training", when he got used to electrical stimulation and increased muscle mass and torque. During that period, he exercised daily with FES for up to one hour and thirty minutes. After that, he did a series of exams to make sure he was able to cycle with FES. These exams analyzed muscle torque, bone density and cardiovascular capacity.

Two protocols were employed. First, there were three trials where the system had to follow a moving reference. It started as a step from 0 instantly to $25 \mathrm{rpm}$. It maintained that cadence for $13 \mathrm{~s}$, and then rose in a ramp up to $42 \mathrm{rpm}$. After $13 \mathrm{~s}$, it lowered to $25 \mathrm{rpm}$, maintained that cadence for $13 \mathrm{~s}$, and then lowered again to rest, always in smooth ramps. These five stages are illustrated on Figure 4. The stimulation current was $40 \mathrm{~mA}$ at $50 \mathrm{~Hz}$, and the pulse width was modulated up to $500 \mu \mathrm{s}$. The second protocol had the participant cycling at a constant cadence of $33 \mathrm{rpm}$. At a specific moment, a disturbance was added to the system simulating a slope. After 20 s, the disturbance was removed. The disturbance applied on this test was a load of $5.9 \mathrm{Kg}$. It was introduced at once, and removed also at once.

On all cases, the stimulation was applied in a trapezoidal shape. In other words, when it was time to stimulate, the pulse width rose from $0 \mu$ s to the specified value by the controller in a ramp. When it was time to stop de stimulation, it also lowered in a ramp. These ramps were set in a manner that right and left leg stimulations overlaped. It has been noticed that such strategy produced smoother movements and, on healthy subjects, more confortable sensations.

\section{RESULTS}

The experimental results obtained during this case study are depicted in Figures 4-7.

During the reference tracking without disturbance experiment, the system followed the reference with an absolute mean error within $2.5 \mathrm{rpm}$ for most of its execution. During the first stage, where the system is supposed to follow the cadence of $25 \mathrm{rpm}$, and on the last one, stopping, the error was larger. On the stopping stage of the second trial there was a much higher error when compared to the other stages and trials. These results are illustrated on Figure 6. Figure 4 represents one of the reference tracking tests results.

On the disturbance experiment, the pre disturbance stage error result was similar to the cadence of $25 \mathrm{rpm}$ from the previous experiment when the absolute mean error is observed, and the post disturbance stage had a slightly better performance. During the disturbance, the error was larger than on the other stages. These results are illustrated on

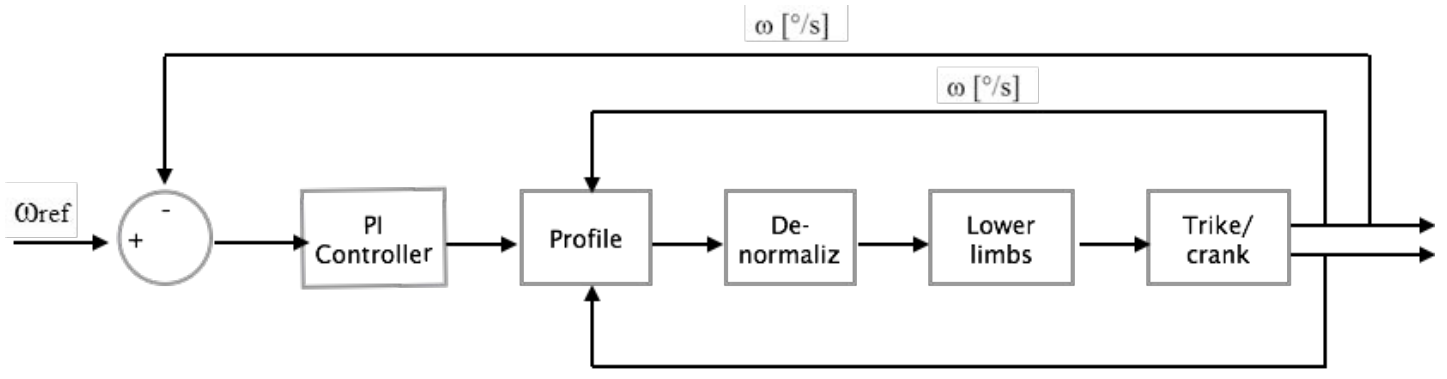

$\Theta\left[{ }^{\circ}\right]$

Figure 3. Block diagram illustrating controller composed by two control loops that computes stimulation timing and intensity. 
Figure 7. Figure 5 represents one of the disturbance rejection tests results.

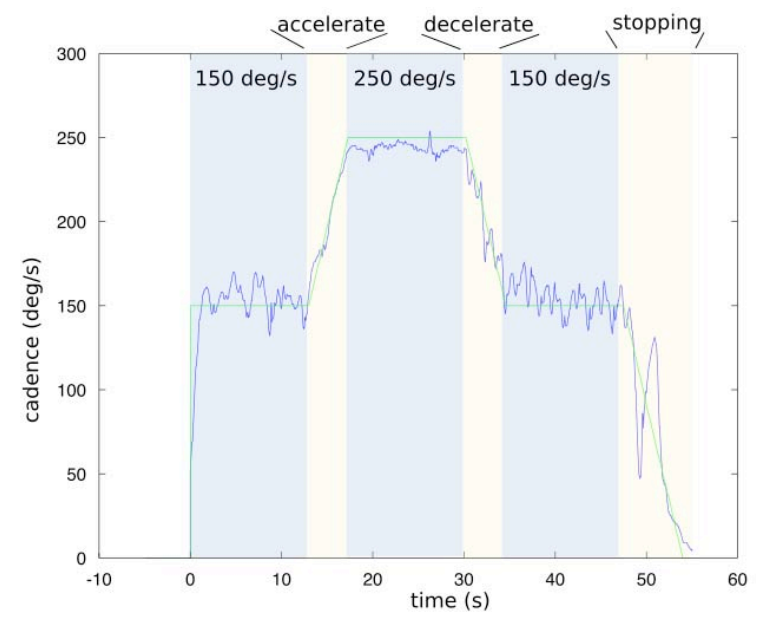

Figure 4. Reference tracking results, trial 1

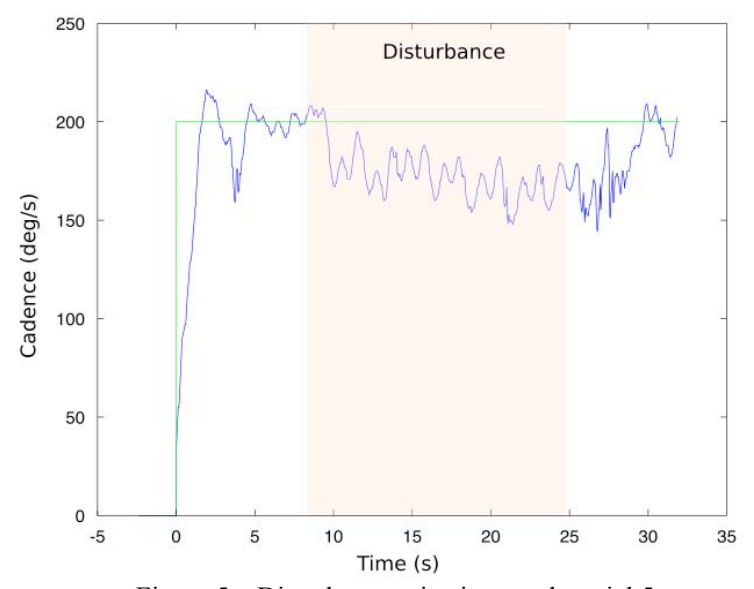

Figure 5. Disturbance rejection results, trial 5

\section{DISCUSSION}

Although this study involved only one SCI subject, the obtained results enable discussing some of the method's advantages and limitations.

During the reference tracking experiment, the target cadence ranged from $25 \mathrm{rpm}$ to $42 \mathrm{rpm}$. The absolute mean error between 0.8 and $2.5 \mathrm{rpm}$ observed on most stages and trials on Figure 6 represents a low error. Since the system stimulates only the quadriceps, the cycling pattern is achieved through pushing the pedals in a controlled timing. However, after an extension of one leg (and flexion of the other), there are no stimuli for the remaining of the movement until it's time for the next leg to extend. For that reason, the crank keeps on rotating only due to the acquired inertia during the previous extension, so the measured cadence on that moment is lower than when the stimulation is taking place. This is visible on Figure 4 by the oscillating aspect of the graph. Every peak is a leg being stimulated, and every valley is the moment when there's no stimulation. Most of the error is due to this effect, which happens also with healthy subjects practicing regular cycling, and should be minimized in order to improve performance.
The reference cadence of $25 \mathrm{rpm}$ was chosen because it is a cadence with which the cycling movement was stable. Below that the participant could get stuck between extensions due to the low inertia. That's why the beginnings of the trials are not ramps, but steps. With an instant step to $25 \mathrm{rpm}$, the controller initial proportional channel is high due to the high error, making the controller start at a high intensity stimulation, achieving the required inertia quickly. As is expected from a PI controller, the cadence takes some time to settle on the reference, making the error values for the first stage higher than the others. The last stage, stopping, however, has reference cadences below $25 \mathrm{rpm}$, which often causes the highest errors.

At the disturbance test, the system wasn't capable of successfully rejecting it. On the other hand, it was able to maintain the cycling pattern, although below the reference cadence. However, if the cadence had dropped below $25 \mathrm{rpm}$, the systems would probably stop, as previously explained. During the time in which the disturbance was applied, the stimulation was saturated, meaning it was requiring maximum effort from the muscles with that current. After the disturbance was removed, the cadence took some time to get back to $33 \mathrm{rpm}$. We suspect it happened because the muscles got slightly fatigued due to the saturation, but quickly recovered and could reach the reference cadence again.

During normal cycling practice, when facing a slope or some kind of resistance, the pilot could lower the gears and would probably be able to keep cycling.

\section{CONCLUSION}

This study case showed that the presented set-up and the developed control strategy is capable of tracking a moving cadence reference for FES cycling, as long as it doesn't drop below $25 \mathrm{rpm}$. However, the cadence oscillates considerably, and this leads us to pursue ways to make it smoother. [7] and other works propose the electrical stimulation of hamstrings and gluteus, along with quadriceps, and a more complex synchronization pattern in order to achieve torque on all crank positions, reducing this oscillating effect.

The disturbance test showed that a disturbance load can

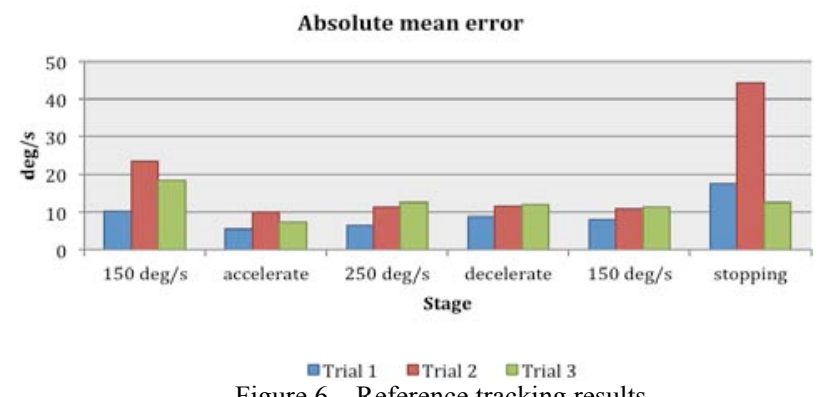

Figure 6. Reference tracking results

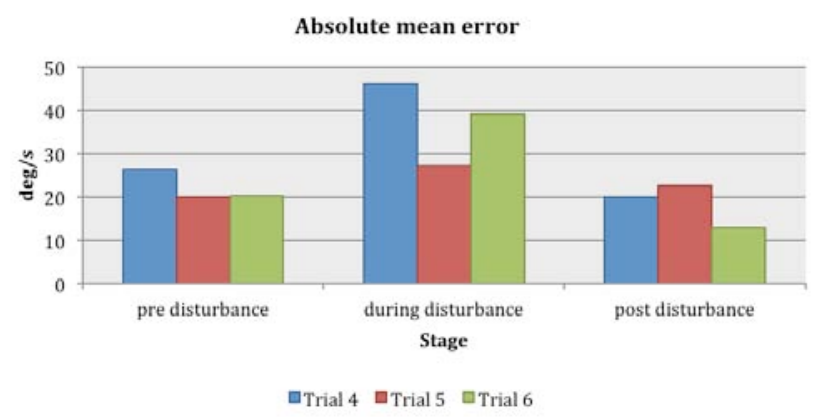

Figure 7. Disturbance rejection results 
affect the cadence in a way that the presented strategy is unable to recover while the load is present. The pilot can keep on cycling and even recover the target cadence if he/she notes it and lower the gears. Therefore, one solution would be to ensure it making this information available, e.g. on a screen. Another solution would be to automate the gears' switching according to the load, crank speed and muscle effort.

The main goal of this work was to evaluate whether our control system was able to reject load perturbations and follow accelerating and decelerating reference cadences. After the tests, some opportunities for improvement were identified. At the same time, our work group is preparing 14 other subjects for further experiments, which will help us analyze our control strategies in comparison to others in the literature. Also, other important variables will be tested, e.g. the influence of SCI chronicity, SCI level, and mechanical aspects.

Besides the stimulation of more muscle groups, future work include the trike's further instrumentation for outdoors cycling. In order to do that, it will have all devices embedded, including the computational unit, electrical stimulator and power sources. Also, brake sensors will be used to stop stimulation during braking to avoid unnecessary effort. Finally, force sensors on both pedals will give precise feedback of each leg torque contribution, allowing the system to independently modulate the stimulation for each one. This should be useful in case one leg is stronger than the other, or fatigues faster.

\section{ACKNOWLEDGMENT}

We would like to thank the participating subject, who continuously motivates us to continue our development effort. We would also like to thank the NTAAI - Núcleo de Tecnologia Assistiva, Acessibilidade e Inovação from FCEUnB.

\section{REFERENCES}

[1] G. M. Davis, N. A. Hamzaid, C. Fornusek. "Cardiorespiratory, metabolic, and biomechanical responses during functional electrical stimulation leg exercise: Health and fitness benefits." Artificial Organs, 32(8):625-629, 2008.

[2] G. Deley, J. Denuziller, N. Babault. "Functional electrical stimulation: Cardiorespiratory adaptations and applications for training in paraplegia." Sports Med, 45(1):71-82, 2014.

[3] S. Mazzoleni, G. Stampacchia, A. Gerini, T. Tombini, and M.C. Carrozza. "Fes-cycling training in spinal cord injured patients." In Engineering in Medicine and Biology Society (EMBC), 2013 35th Annual International Conference of the IEEE, pages 5339-5341, July 2013.

[4] M. Kjaer, G. Perko, N. H. Secher, R. Boushel, N. Beyer, S. Pollack, A. Horn, A. Fernandes, T. Mohr, S. F. Lewis, and H. Galbo. "Cardiovascular and ventilatory responses to electrically induced cycling with complete epidural anaesthesia in humans." Acta Physiol. Scand., 151:199-207, 1994.

[5] R. Downey, E. Ambrosini, S. Ferrante, A. Pedrocchi, W. Dixon, G. Ferrigno. "Asynchronous Stimulation With An Electrode Array Reduces NMES-Induced Muscle Fatigue During FES Cycling.” IFESS 2012.

[6] A. P. L. Bó, L. O. Fonseca, S. R. Rodrigues, A. C. C. De Sousa, L. L. Oliveira, and G. A. Brindeiro. "Experiments on Lower Limbs FES Control for Cycling." SBAI 2015.

[7] K. J. Hunt. "Control Systems for Function Restoration, Exercise, Fitness and Health in Spinal Cord Injury", PhD thesis, University of Glasgow, 2005. 